\title{
Plain and contrast-enhanced chest computed tomography scan findings of pulmonary cryptococcosis in immunocompetent patients
}

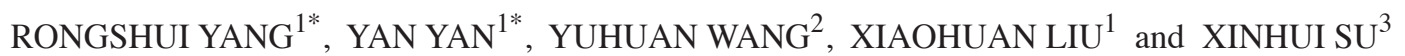 \\ ${ }^{1}$ Department of Radiology, Xinglin Branch Hospital, The First Affiliated Hospital of Xiamen University; \\ ${ }^{2}$ Department of Pathology, The First Affiliated Hospital of Xiamen University, Xiamen, Fujian 361003; \\ ${ }^{3}$ Department of Nuclear Medicine, Zhongshan Hospital, Xiamen University, Xiamen, Fujian 361004, P.R. China
}

Received November 4, 2016; Accepted June 23, 2017

DOI: $10.3892 /$ etm.2017.5096

\begin{abstract}
Pulmonary cryptococcosis is most commonly reported in immunocompromised patients, whereas immunocompetent hosts are rarely affected and may be asymptomatic, resulting in reduced diagnostic performance of computed tomography (CT) imaging. Thus, the aim of the present study was to review the plain and contrast-enhanced chest CT scan findings of primary pulmonary cryptococcosis in immunocompetent patients, with the aim of improving the diagnosis of this type of pulmonary disease. In the present study, a total of 27 immunocompetent patients of clinically confirmed pulmonary cryptococcosis were analyzed retrospectively. Of the 27 patients, 14 patients underwent plain and contrast-enhanced chest $\mathrm{CT}$ scans, while 13 patients only underwent plain chest $\mathrm{CT}$ scanning. The clinical and imaging characteristics, including the location, shape, size, number, edge and attenuation or intensity of each lesion, in unenhanced and contrast-enhanced CT scans were reviewed. The results indicated that the most common CT finding was pulmonary nodules $(40.74 \%)$, with multiple nodules (25.93\%) being more common compared with solitary nodules $(14.81 \%)$. The majority of the nodules were poorly defined and inhomogeneous with observed air-bubble sign. Other findings included consolidation (25.93\%), ground-glass opacities (GGO; 22.22\%) and a mass (11.11\%). The halo, air bronchogram and cavity signs were observed more frequently $(22.22,18.52$ and $14.81 \%$, respectively). The pulmonary lesions presented a predominant distribution in the lower lung lobes and peripheral area in 55.55 and $74.07 \%$ of
\end{abstract}

Correspondence to: Dr Xinhui Su, Department of Nuclear Medicine, Zhongshan Hospital, Xiamen University, 201 Hubin South Road, Xiamen, Fujian 361004, P.R. China

E-mail: suxinhuii@163.com

${ }^{*}$ Contributed equally

Key words: pulmonary cryptococcosis, immunocompetent patients, computed tomography features, diagnosis the cases, respectively. On the contrast-enhanced CT images, the majority of nodules presented ring enhancement with the mean maximal enhancement value of $20.92 \pm 5.67 \mathrm{Hu}$, and masses demonstrated inhomogeneous enhancement with a mean maximal enhancement value of $35.61 \pm 8.32 \mathrm{Hu}$. In conclusion, familiarity with the CT findings and occupational environment exposure history will assist in earlier and easier diagnosis of pulmonary cryptococcosis in immunocompetent patients.

\section{Introduction}

Cryptococcosis is a potentially life-threatening fungal disease affecting humans and animals worldwide. The disease has increased in prevalence in recent years, accounting for 1 million infections and 700,000 deaths annually $(1,2)$. In China, cryptococcosis has also gradually increased over the past 20 years (3). The majority of these infection cases are due to the inhalation of Cryptococcus spores existing in pigeon excreta and decayed wood. Among $>38$ species of the genus Cryptococcus, Cryptococcus neoformans and Cryptococcus gattii are the major causative agents of human cryptococcosis (2). These two species are genetically associated with each other; however, they differ genotypically, epidemiologically, ecologically, geographically and phenotypically $(2,3)$. Cryptococcus neoformans occurs most frequently in immunocompromised individuals, particularly those with acquired immunodeficiency syndrome (AIDS) or other causes of impaired T cell-mediated immunity, and less frequently infects immunocompetent patients (4). By contrast, Cryptococcus gattii is more likely to infect healthy individuals, and is associated with tropical and subtropical regions (5). In addition, Cryptococcus gattii infections cause more frequent neurological complications as compared with infections associated with Cryptococcus neoformans. Infections with Cryptococcus gattii are also more difficult to treat due to its reduced sensitivity to antifungal therapy in comparison with Cryptococcus neoformans; therefore, more aggressive therapeutic strategies are usually required for these infections $(6,7)$.

Pulmonary cryptococcosis may be the first manifestation of cryptococcosis since the respiratory tract is considered 
to be the entry site and is most frequently involved when cryptococcal infection develops (8). Depending on the host immune status, the infection may remain dormant in the lung or may undergo hematogenous spread to any organ system, including the central nervous system (CNS), bones and skin $(7,9)$. However, pulmonary cryptococcosis does not present specific clinical manifestations and radiological findings. The clinical expression is variable and may present as coughing, expectoration, chest tightness, fever or asymptomatic. The most common radiological characteristics are known to be solitary or multiple pulmonary nodules, and segmental or lobar consolidation $(10,11)$. For these reasons, pulmonary cryptococcosis is frequently misdiagnosed as other lung diseases, such as cancer and pneumonia. Thus, the main diagnostic methods include culture of clinical samples, detection of cryptococcal antigen in body fluids and/or histopathological examinations in tissue sections (12). However, certain of these methods are associated with invasive acquisition of biopsies from the patients. In addition, the sensitivities and accuracies of these techniques are limited due to sampling errors or pollution, and heterogeneity of Cryptococcus spores in biopsy samples $(13,14)$. Computed tomography (CT) scanning allows a quantitative repeated noninvasive evaluation of the overall course of pulmonary disease in patients. Therefore, a chest CT scan is helpful for identification of pulmonary disease, patient stratification, prediction of the response to therapy and evaluation of drug efficacy. Although there are certain studies in the literature reporting plain chest $\mathrm{CT}$ imaging data of pulmonary cryptococcosis $(4,10,11)$, poorly specific manifestations in plain chest CT scans often cause misdiagnosis. Contrast-enhanced chest CT scans can provide more valuable information for the diagnosis of pulmonary nodules in comparison with plain chest CT scans (15). Thus, the aim of the present study was to retrospectively review the finding of plain and contrast-enhanced chest CT scans in immunocompetent patients with pulmonary cryptococcosis, in order to improve the diagnosis of this type of pulmonary disease.

\section{Materials and methods}

Patients. The current study retrospectively reviewed the characteristics and imaging findings of 27 immunocompetent patients diagnosed with pulmonary cryptococcosis who were admitted to Xinglin Branch, the First Affiliated Hospital of Xiamen University (Xiamen, China) and underwent chest CT examination (plain and contrast-enhanced, or only plain) between January 2005 and October 2014. The patient's medical records were collected, including demographics, respiratory symptoms, occupational environment exposure history, physical examination results, laboratory tests, imaging data, pathology data, treatment and outcomes. The diagnosis of pulmonary cryptococcosis was confirmed by histological or cytological presence of the organism in lung biopsy specimens, by positive findings in respiratory specimen cultures or in the serum cryptococcal antigen test The protocol was approval by the Ethics Committee of Xinglin Branch Hospital and all patients or legally authorized representatives provided written informed consent upon enrolment into the present study.
Chest CT scanning. Of the 27 patients, 14 patients underwent plain and contrast-enhanced chest CT scans, and 13 patients only underwent plain chest CT scans using a Philips Brilliance 16 CT scanner (Philips Medical Systems, Inc., Cleveland, OH, USA) or a Toshiba Aquilion 16 CT scanner (Toshiba Medical Systems Corp., Tokyo, Japan). Patients in the supine position were asked to hold their breath during scanning. Scanning was performed from the lung apices to the level of the middle portion of the two kidneys. The CT images were obtained using the following parameters: Detector collimation, $5 \mathrm{~mm}$; tube voltage, $120 \mathrm{kV}$; tube current, 100-200 mA (using an automatic exposure control system); and reconstruction interval, $5 \mathrm{~mm}$. Contrast-enhanced CT scans were conducted by administration of a nonionic iodine contrast agent (Iopromide 300; Bayer Schering Pharma AG, Leverkusen, Germany). Patients were initially fast injected with Iopromide 300 intravenously at the dose of $1.5 \mathrm{ml} / \mathrm{kg}$ and speed of 3-4 ml/sec with a high-pressure syringe, and then artery and vein double-phase scans were performed with automatically triggered technology. All images were processed in a picture archiving and communication system (PACS; version UnSight 4.2; DJ HealthUnion Systems Corp., Shanghai, China) for coronal, sagittal and oblique reconstructions.

Image analysis. CT images were reviewed retrospectively by two independent experienced radiologists who were unaware of any clinical data. A final conclusion was reached by consensus. When CT images were reviewed, the size, number, location, border and internal characteristics of any pulmonary nodules or masses were recorded. The presence of an air bubble, air bronchogram, halo sign, cavity, calcification and pleural effusions was also noted. The CT attenuation values of the nodule or mass were subsequently measured in the largest parts of the lesions, which were away from the air density area. The mean attenuation values were calculated from three randomly selected nodule locations.

\section{Results}

Patient characteristics. The demographics and clinical features of the 27 immunocompetent patients with pulmonary cryptococcosis included into the present study are summarized in Table I. These patients consisted of 16 (59.26\%) men and $11(40.74 \%)$ women, with an age range between 26 and 65 years (median, 45.6 years). The most common clinical manifestations were coughing (9 cases; 33.33\%), expectoration (6 cases; $22.22 \%$ ), fever (5 cases; $18.52 \%$ ) and chest pain (4 cases; $14.81 \%$ ), while $3(11.11 \%$ ) patients were asymptomatic. In term of disease history, 8 (29.63\%) patients were healthy, whereas $7(25.93 \%)$ patients had a history of chronic pulmonary disease, $6(22.22 \%)$ diabetes, $5(18.52 \%)$ hypertension and $1(3.70 \%)$ malignancy. Regarding environmental factors, $8(29.63 \%)$ patients had no exposure history, while $9(33.33 \%)$ patients had a history of direct exposure to pigeon droppings. The remaining $10(37.04 \%)$ patients have a history of keeping cats, dogs or poultry.

According to the results of laboratory examination (Table II), elevation of the peripheral white blood cell count was detected in $8(29.63 \%)$ patients (normal range, $3.5-9.5 \times 10^{-9}$ cells $\left./ 1\right)$. A lymphocyte cell count of $<1.1 \times 10^{-9} / 1$ 
Table I. Demographics and clinical features of 27 immunocompetent patients with pulmonary cryptococcosis.

\begin{tabular}{|c|c|}
\hline Characteristics & Subjects \\
\hline \multicolumn{2}{|l|}{ Age, years } \\
\hline Range & $26-65$ \\
\hline Mean & 45.6 \\
\hline \multicolumn{2}{|l|}{ Gender, n (\%) } \\
\hline Male & $16(59.26)$ \\
\hline Female & $11(40.74)$ \\
\hline \multicolumn{2}{|l|}{ Symptoms, n (\%) } \\
\hline Asymptomatic & $3(11.11)$ \\
\hline Coughing & $9(33.33)$ \\
\hline Expectoration & $6(22.22)$ \\
\hline Fever & $5(18.52)$ \\
\hline Chest pain & $4(14.81)$ \\
\hline \multicolumn{2}{|l|}{ Disease history, n (\%) } \\
\hline Healthy & $8(29.63)$ \\
\hline Chronic pulmonary disease & $7(25.93)$ \\
\hline Diabetes & $6(22.22)$ \\
\hline Hypertension & $5(18.52)$ \\
\hline Malignancy & $1(3.70)$ \\
\hline \multicolumn{2}{|l|}{$\begin{array}{l}\text { Occupational environment exposure } \\
\text { history, } \mathrm{n}(\%)\end{array}$} \\
\hline History of exposure to pigeon droppings & $9(33.33)$ \\
\hline History of keeping cats, dogs or poultry & $10(37.04)$ \\
\hline No exposure history & $8(29.63)$ \\
\hline \multicolumn{2}{|l|}{ Treatment and follow-up, n (\%) } \\
\hline No treatment & $4(14.81)$ \\
\hline Antifungal drugs & $23(85.19)$ \\
\hline \multicolumn{2}{|l|}{ Diagnostic method, n (\%) } \\
\hline Lung biopsy specimens & $12(44.44)$ \\
\hline $\begin{array}{l}\text { Positive findings in respiratory } \\
\text { specimen culture }\end{array}$ & $10(37.04)$ \\
\hline $\begin{array}{l}\text { Positive results of serum cryptococcal } \\
\text { antigen test }\end{array}$ & $5(18.52)$ \\
\hline
\end{tabular}

Values are expressed as the number $(\%)$

(normal range, $1.1-3.2 \times 10^{-9}$ cells/l) was present in $4(14.81 \%)$ patients (3 with chronic pulmonary disease receiving corticosteroids and 1 with malignancy), and a hemoglobin level of $<115 \mathrm{~g} / 1$ (normal range, 115-150 g/l) was observed in $5(18.52 \%)$ patients ( 2 with diabetes, 1 with malignancy and 2 with chronic pulmonary disease receiving corticosteroids). In addition, an erythrocyte sedimentation rate of $>15 \mathrm{~mm} / \mathrm{h}$ (normal range, $0-15 \mathrm{~mm} / \mathrm{h}$ ) was reported in $2(7.41 \%)$ male patients and $>20 \mathrm{~mm} / \mathrm{h}$ (normal range, $0-20 \mathrm{~mm} / \mathrm{h}$ ) in $4(22.22 \%$ ) female patients (4 with chronic pulmonary disease and 2 with hypertension), while a c-reactive protein level of $>8 \mathrm{mg} / \mathrm{l}$ (normal range, $0-8 \mathrm{mg} / \mathrm{l}$ ) was observed in $5(18.52 \%)$ patients (3 with chronic pulmonary disease and 2 with diabetes). None of the patients presented CNS involvement of the disease.
Table II. Laboratory test of 27 immunocompetent patients with pulmonary cryptococcosis.

\begin{tabular}{lccc}
\hline Parameters & Unit & $\begin{array}{c}\text { Normal } \\
\text { range }\end{array}$ & $\begin{array}{c}\text { Abnormal, } \\
\mathrm{n}(\%)\end{array}$ \\
\hline White blood cells & $\times 10^{-9} / 1$ & $3.5-9.5$ & $8(29.63)$ \\
Lymphocytes & $\times 10^{-9} / 1$ & $1.1-3.2$ & $4(14.81)$ \\
Hemoglobin & $\mathrm{g} / 1$ & $115-150$ & $5(18.52)$ \\
Platelet & $\times 10^{-9} / 1$ & $125-350$ & 0 \\
ESR of males & $\mathrm{mm} / \mathrm{H}$ & $0-15$ & $2(7.41)$ \\
ESR of females & $\mathrm{mm} / \mathrm{H}$ & $0-20$ & $4(22.22)$ \\
C-reactive protein & $\mathrm{mg} / 1$ & $0-8$ & $5(18.52)$ \\
\hline
\end{tabular}

Values are expressed as the number (\%). ESR, erythrocyte sedimentation rate.

The diagnosis of these pulmonary cryptococcosis cases was confirmed by histological or cytological presence of the organism in lung biopsy specimens (12 cases; $44.44 \%$ ), by positive findings in respiratory specimen culture (10 cases; $37.04 \%$ ) or by positive results of the serum cryptococcal antigen test (5 cases; 18.52\%). Treatment included antifungal drugs alone in $23(85.19 \%)$ patients, and no treatment was administered in $4(14.81 \%)$ patients, one of whom suffered from pancreatic cancer that progressed rapidly, and thus antifungal treatment was impossible. Of the 23 patients who received antifungal therapy, 22 patients improved and 1 succumbed due to acute myocardial infarction during the follow-up period.

Radiologicalfeatures. The pulmonary abnormalities observed on the CT scans are summarized in Table III. The most common radiologic feature of pulmonary crptococcosis was the presence of one or more nodules (11 out of 27 patients; $40.74 \%$ ). A solitary nodule was identified in $36.36 \%$ (4 out of 11) of patients (Fig. 1), while multiple nodules were observed in $63.64 \%$ (7 out of 11) of patients and were most commonly bilateral in $71.43 \%$ ( 5 out of 7 ) of these patients. The nodules were $6-20 \mathrm{~mm}$ in diameter in 6 patients and $21-30 \mathrm{~mm}$ in 5 patients. The majority of the nodules had poorly defined margins, with peripheral predominance (outer third of the lung) close to the pleura in $72.73 \%$ ( 8 out of 11 ) of patients. The second most common finding was consolidation ( 7 out of 27 patients; 25.93\%; Fig. 2). Other associated findings included ground-glass opacities (GGO; 6 out of $27 ; 22.22 \%$ ) and presence of a mass (3 out of 27; 11.11\%; Fig. 3 ).

Lesions in the right lung were observed in 10 (37.04\%) patients and in the left lung in $10(37.04 \%)$ patients, while bilateral lung lesions were observed in $7(25.93 \%)$ patients. The lower lobes were most frequently involved in $15(55.55 \%)$ patients, 7 (25.93\%) patients had lesions involvement in the upper lobes and $5(18.52 \%)$ patients had middle lobe involvement. Furthermore, lesions in the peripheral area were identified in $20(74.07 \%)$ patients and in the central area in $7(25.93 \%)$ patients. These data suggested that the pulmonary lesions presented lower lung lobe and peripheral predominance.

Air-bubble sign was a feature in the CT scans of $6(54.55 \%)$ patients with a lung nodule (Fig. 1A), 2 (33.33\%) patients with 
Table III. Radiological features of pulmonary abnormalities of CT scans.

\begin{tabular}{|c|c|c|c|c|c|}
\hline Characteristics & Nodule & Consolidation & GGO & Mass & Total no. $(\%)$ \\
\hline Patient no. (\%) & $11(40.74)$ & $7(25.93)$ & $6(22.22)$ & $3(11.11)$ & 27 \\
\hline \multicolumn{6}{|l|}{ Feature number } \\
\hline Solitary & 4 & 2 & 2 & 3 & $11(40.74)$ \\
\hline Multiple & 7 & 5 & 4 & 0 & $16(59.26)$ \\
\hline \multicolumn{6}{|l|}{ Lesion lung } \\
\hline Right lung & 2 & 3 & 4 & 1 & $10(37.04)$ \\
\hline Left lung & 4 & 2 & 2 & 2 & $10(37.04)$ \\
\hline Bilateral lungs & 5 & 2 & 0 & 0 & 7 (25.92) \\
\hline \multicolumn{6}{|l|}{ Lung area } \\
\hline Upper lobes & 3 & 2 & 2 & 0 & $7(25.93)$ \\
\hline Middle lobe & 2 & 1 & 1 & 1 & $5(18.52)$ \\
\hline Lower lobes & 6 & 4 & 3 & 2 & $15(55.55)$ \\
\hline \multicolumn{6}{|l|}{ Predominant distribution } \\
\hline Central & 3 & 2 & 1 & 1 & $7(25.93)$ \\
\hline Peripheral & 8 & 5 & 5 & 2 & $20(74.07)$ \\
\hline \multicolumn{6}{|l|}{ Margin } \\
\hline Poorly defined & 7 & 7 & 4 & 2 & $20(74.07)$ \\
\hline Smooth & 4 & 0 & 2 & 1 & $7(25.93)$ \\
\hline Halo sign & 6 & 0 & 0 & 0 & $6(22.22)$ \\
\hline Lobulated & 1 & 0 & 0 & 2 & $1(3.70)$ \\
\hline Spiculated & 1 & 0 & 0 & 1 & $1(3.70)$ \\
\hline \multicolumn{6}{|l|}{ Internal characteristics } \\
\hline Air-bubble sign & 6 & 0 & 2 & 1 & $9(33.33)$ \\
\hline Air bronchogram & 0 & 5 & 0 & 0 & $5(18.52)$ \\
\hline Cavity & 0 & 2 & 0 & 2 & $4(14.81)$ \\
\hline Calcification & 1 & 0 & 0 & 0 & $1(3.71)$ \\
\hline Homogenous & 4 & 0 & 4 & 0 & $8(29.63)$ \\
\hline \multicolumn{6}{|c|}{ Concurrent characteristics } \\
\hline Pleural effusion & 0 & 2 & 0 & 0 & $2(7.41)$ \\
\hline Lymph nodes $^{\mathrm{a}}$ & 0 & 3 & 0 & 1 & $4(14.81)$ \\
\hline None & 11 & 2 & 6 & 2 & $21(77.78)$ \\
\hline \multicolumn{6}{|l|}{ Maximal $\mathrm{CT}$ value $(\mathrm{Hu})$} \\
\hline Enhancement value & $20.92 \pm 5.67$ & - & - & $35.61 \pm 8.32$ & - \\
\hline Lesion enhancement & Ring & - & - & Inhomogeneous & - \\
\hline
\end{tabular}

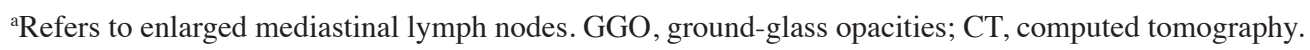

GGO and 1 patient with a mass. Nodules with calcification or halo sign were observed in $1(9.1 \%)$ or $6(54.55 \%)$ patients, respectively. Air bronchogram sign (Fig. 2A) was identified in $5(71.43 \%)$ patients with a consolidation lesion, while a cavity was noted in $2(28.57 \%)$ patients with consolidation (Fig. 2A) and in 2 patients with a mass lesion (Fig. 3A). In addition, mediastinal lymph node enlargement was noted in 3 patients with a consolidation lesion and in 1 patient with a mass lesion. Pleural effusion was noted in 2 patients with a consolidation lesion, however, pericardial effusion was not observed in any of the patients. Thus, the air-bubble, halo, air bronchogram and cavity signs were the most commonly reported features in $\mathrm{CT}$ scans $(33.33,22.22,18.52$ and $14.81 \%$, respectively).
Out of the 27 participants, 11 patients with a nodule lesion and 3 patients with mass lesions also underwent CT contrast-enhancement evaluation. For the patients with nodule lesions, the nodules presented ring enhancement with a mean maximal enhancement value of $20.92 \pm 5.67 \mathrm{Hu}$ (Fig. 3B). Among the 3 patients with mass lesions, the lesions exhibited inhomogeneous enhancement with a mean maximal enhancement value of $35.61 \pm 8.32 \mathrm{Hu}$ (Fig. 3B).

Follow-up studies. The range of follow-up for the $27 \mathrm{immu-}$ nocompetent patients was 1-24 months (mean, 13.2 months). During the follow-up period, no mortalities were reported as a result of pulmonary cryptococcosis. The clinical or radiological characteristics were significantly improved in 26 patients 

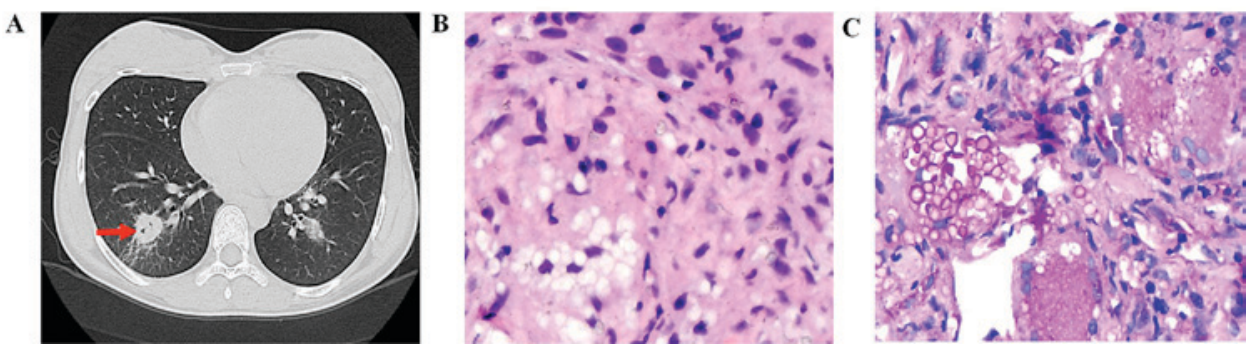

Figure 1. Representative case of a 26-year-old immunocompetent woman with pulmonary cryptococcosis. (A) CT imaging in the lung window demonstrated a nodule with poorly defined margins and air-bubble sign in the lower lobes of bilateral lungs. (B) Histological staining of the specimen of CT-guided percutaneous transthoracic needle biopsy in the right lower lobe. Hematoxylin and eosin staining (magnification, x400) exhibited granulomatous inflammation. (C) Periodic acid-Schiff staining (magnification, x400) identified Cryptococcus spores in the cytosol and interstitial spaces. Red arrow indicates a nodule with poorly defined margins and air-bubble signs in the right lower lobes. CT, computed tomography.
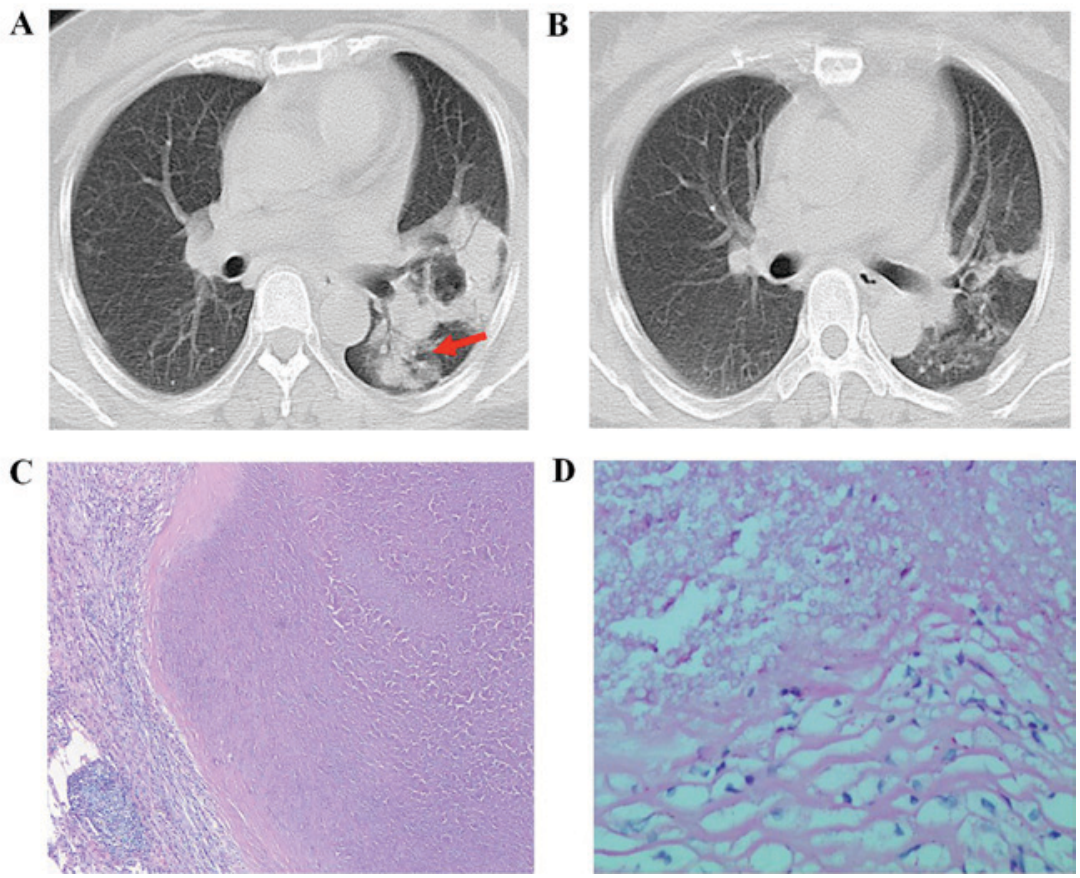

Figure 2. Representative case of a 48-year-old immunocompetent woman with pulmonary cryptococcosis.(A) CT imaging demonstrated a large consolidation with cavity and air bronchograms in the left lower lobe. (B) At 3-month follow-up following antifungal therapy, the CT scan demonstrated that the consolidation had been evidently resolved, and the signs of cavity and air bronchogram had disappeared.(C) Specimen obtained by percutaneous transthoracic needle biopsy in the left lower lobe revealed epithelioid granulomatous inflammation with focal necrosis (hematoxylin and eosin staining; magnification, x200). (D) Periodic acid-Schiff staining (magnification, $\mathrm{x} 400$ )demonstratedCryptococcussporesinthefocalnecrosis.Redarrowindicatesaconsolidation withcavity andairbronchogramsintheleftlowerlobe. CT, computed tomography.

(96.30\%). In 1 patient (3.70\%) who received no antifungal treatment due to rapid progression of pancreatic cancer, the pulmonary lesions of multiple nodules demonstrated disease progression over a 3-month follow-up period. After a short time, the patient succumbed to pancreatic cancer.

At the 3-month follow-up evaluation of the 23 patients who received antifungal therapy only, 16 patients exhibited partial resolution of pulmonary abnormalities with a decrease in the titer of serum cryptococcal antigen test or the culture of respiratory specimens (Fig. 2), whereas the condition of 5 patients remained unchanged, and 2 patients presented radiographic deterioration and were re-treated with antifungal therapy. At the 6- and 9-month follow-up evaluation, 20 patients demonstrated radiographic improvement and 3 patients remained radiographically stable with a decrease in the titer of serum cryptococcal antigen or the culture of respiratory specimens. At the $>12$ months of follow-up, the radiographs in all 23 patients presented significant resolution of pulmonary abnormalities with a parallel decrease in the titer of serum cryptococcal antigen or the culture of respiratory specimens.

At the 3- and 6-month follow-up evaluation of 3 patients without any therapy, CT revealed reduction in the size of the parenchymal abnormalities. At the 9-month follow-up, the lung lesions disappeared completely.

\section{Discussion}

Pulmonary cryptococcosis is an important opportunistic fungal infection, with the majority of cases caused by Cryptococcus neoformans or Cryptococcus gattii infection $(1,2)$. Epidemiological data indicate that 
A

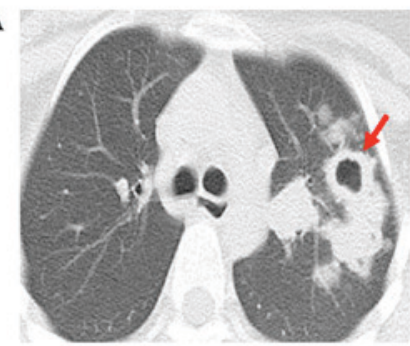

D

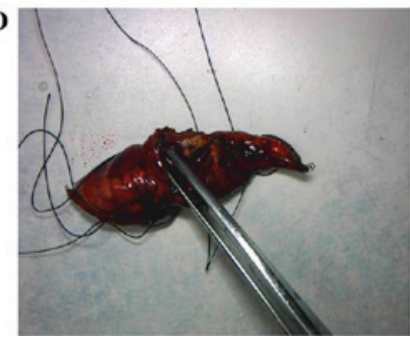

B

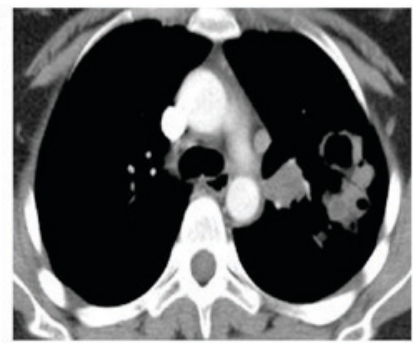

E

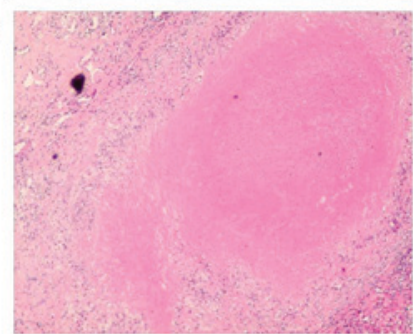

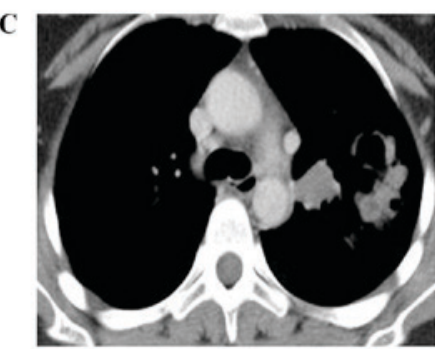

F

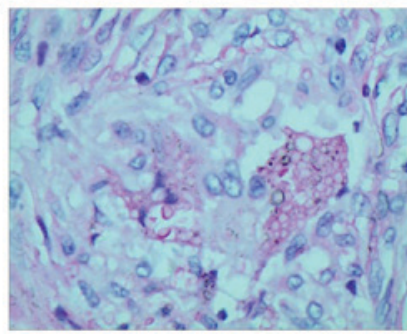

Figure 3. Representative case of a 38-year-old immunocompetent man with pulmonary cryptococcosis. (A) CT imaging in the lung window demonstrated a nodule with a clear boundary. (B) Artery phase contrast-enhanced chest CT scan in the mediastinal window indicated that the nodule and mass were not evidently enhanced. (C) Vein phase contrast-enhanced chest CT scan in the mediastinal window indicated that the nodule presented ring enhancement with a maximal enhancement value of $24.25 \mathrm{Hu}$, and the mass presented inhomogeneous enhancement with a maximal enhancement value of $39.21 \mathrm{Hu}$. (D) Resected specimen of the left upper lobe. (E) Hematoxylin and eosin staining (magnification, x100) exhibited epithelioid granulomatous lesions with focal necrosis, and Cryptococcus spores were vaguely observed. (F) Periodic acid-Schiff staining (magnification, $\mathrm{x} 400$ ) demonstrated the Cryptococcus spores in macrophages Red arrow indicates a nodule with a clear boundary and short spiculated with lobulated, cavity and air-bubbles in the left upper lobe. CT, computed tomography.

Cryptococcus neoformans occurs more frequently in immunocompromised individuals, particularly those with AIDS. By contrast, Cryptococcus gattii is more likely to cause disease in healthy people (2), as recently highlighted in the outbreaks in Canada (British Columbia) and the United States Pacific Northwest (1). However, Cryptococcus gattii infections are rare in China. Feng et al (16) reported that only 9 out of $110(8.2 \%)$ clinical Cryptococcus isolates from China were Cryptococcus gattii, and the vast majority were Cryptococcus neoformans, which was consistent with the data of several studies of clinical molecular epidemiology in other geographic areas $(12,17)$.

Previously, pulmonary cryptococcosis was considered to occur mainly in immunocompromised individuals, such as individuals with AIDS, as well as in individuals that underwent organ transplantation or had a hematologic malignancy, and to be rare in immunocompetent patients (4). Recently, it has reported that the incidence of pulmonary cryptococcosis is increasing (12). A epidemiological study indicated that the annual incidence of pulmonary cryptococcosis has increased from 6 cases per million in 1999 to 38 cases per million in 2006 in British Columbia, Canada (18). This $>6$-fold increase cannot be explained by the stable incidence of people with human immunodeficiency virus (HIV) infection. Thus, the increased incidence is mainly due to infection of non-HIV (immunocompetent) individuals $(19,20)$.

Studies on the prevalence and epidemiology of pulmonary cryptococcosis in China are relatively scarce. A recent systemic review by Yuchong et al (21) summarized 1,032 reports of 8,769 cases of pulmonary cryptococcosis in mainland China between 1985 and 2010. Notably, these rates were similar to those reported in Taiwan, where the average annual incidence increased from 4.0 cases per million individuals in 2000 to 5.5 per million individuals in 2007 (22). Considering the aforementioned studies, it is evident that pulmonary cryptococcosis cases in China have been increasing over the recent decades $(19,21,23)$. In the present study of cases at a single-institution, the retrospective review of $27 \mathrm{immu}-$ nocompetent patients clinically confirmed with pulmonary cryptococcosis revealed that pulmonary cryptococcosis is not rare in immunocompetent people. In these patients, the disease did not have specific clinical manifestations and radiological findings, and the clinical prognosis was most often favorable. The majority of patients in the current study presented with respiratory symptoms, including coughing (33.33\%), expectoration $(22.22 \%)$, fever $(18.52 \%)$ and chest pain $(14.81 \%)$, while only 3 patients $(11.11 \%)$ were asymptomatic. In contrast to the present study, Kishi et al (10) reported that approximately one-third of immunocompetent patients with pulmonary cryptococcosis were asymptomatic. Depending on the host immune status, cryptococcosis may disseminate from the lungs to other organs, including the CNS and brain. One of the proposed mechanisms for cryptococcosis crossing the blood-brain barrier is by hiding inside mononuclear phagocytes via a 'Trojan horse' mechanism $(24,25)$. Thus, besides pulmonary cryptococcosis, Cryptococcus infection can be frequently associated with cryptococcal meningoencephalitis, resulting in high mortality and morbidity rates. No patients in the current study presented with cryptococcal meningoencephalitis. Compared with immunocompetent patients, the symptoms of cryptococcosis infection in immunocompromised patients are more evident $(19,20,23)$.

Pulmonary cryptococcosis appears to predominantly occur in adult males. In the current study, the 27 patients included 16 males and 11 females. The male: female ratio (1.45:1) in this group is similar to reported in previous studies $(23,26)$. Furthermore, in the present study, 33.33\% (9 out of 27) of patients had a history of direct exposure to pigeon droppings and $37.04 \%$ (10 out of 27) of patient had a history of keeping cats, dogs or poultry. Only $29.63 \%$ (8 out of 27 ) patients had no exposure history. These data suggest that more attention should 
be paid to detailed questioning regarding the occupational and environmental exposure history of patients in the diagnosis of pulmonary cryptococcosis.

The radiological findings of pulmonary cryptococcosis in plain chest CT scans are diverse. In the present study, the most common manifestation of pulmonary cryptococcosis in plain chest CT scans was pulmonary nodules, including single and multiple nodules, which were observed in $40.74 \%$ (11 out of 27 ) of patients, in comparison with $46.06 \%$ of patients in the study by Ye et al (27) and 55.2\% in the study by Zhang et al (23). Multiple nodules $(63.64 \%)$ were more common compared with single nodules $(36.36 \%)$ and bilateral lung lesions were identified in certain patients $(45.45 \%)$, consistent with previous findings (19). The margin of the nodules can be ill-defined, smooth, lobulated or spiculated. In the present study, the majority (63.64\%) of the nodules in the patients had poorly defined margins, with $54.55 \%$ (6 out of 11 patients) of the nodules presenting a halo sign, while only $9.09 \%$ (1 out of 11 patients) of the nodules were lobulated or speculated, which were misdiagnosed as lung cancer. Lee et al (26) reported that none of the nodules of $12 \mathrm{immu}-$ nocompromised and immunocompetent patients demonstrated a smooth margin. Furthermore, Lindell et al (28) reported that $77.0 \%$ (7 out of 10 immunocompetent patients) of the nodules were well-defined with smooth margins, and Zinck et al (29) demonstrated that margination of nodules in the reported cases was smooth $(50.0 \%$; $n=5)$, poorly defined $(30.0 \% ; n=3)$, lobulated $(10.0 \% ; n=1)$ or speculated $(10.0 \% ; n=1)$ in 10 patients with immunocompromised or immunocompetent. The halo sign is caused by various pathological conditions, including hemorrhagic nodules of varying causes, tumor cell infiltration and non-hemorrhagic inflammatory lesions (30). This sign in patients with pulmonary cryptococcosis has been demonstrated to occur in association with granulomatous inflammatory (20).

Other CT findings in the present study included consolidation, GGO and a mass, with 7, 6 and 3 cases identified, respectively. Internal characteristics in the nodules, consolidation, GGO and mass lesions included air bubble (33.33\%), air bronchogram (18.52\%), cavity (14.81\%) and calcification $(3.7 \%)$. The air-bubble sign was observed in 6 patients with a lung nodule lesion due to cryptococcosis erosion of a bronchiolus allowing air to reach the space between granulomatous lesions. In addition, air bronchogram was observed in 5 out of 7 patients with consolidation due to inflammatory response composed of histiocytes, multinucleated giant cells and lymphocytic infiltration without destruction of tissue architectures (20). Cavitation was also evident on CT scans in $4(14.81 \%)$ of the 27 patients, including cavitating consolidation in 2 patients and cavitating mass in two, which were difficult to be distinguished from lung cancer and tuberculosis. These aforementioned findings were similar to those of previous studies $(23,28,29)$. Pulmonary cavitation has been more rarely reported in immunocompetent patients compared with immunocompromised patients (10). It has also been reported that cavitation demonstrates a long-term localized pulmonary abnormality, indicating that patients with cavitary pulmonary lesions may experience a more severe cryptococcal infection that requires a more aggressive antifungal therapy (31).

Regarding the distribution of the lesions of pulmonary cryptococcosis, in agreement with prior radiographic studies $(15,23,28,30)$, the pulmonary lesions in the present study were located predominantly in the lower lung (55.55\%) rather than in the middle (18.52\%) or upper lung (25.52\%), and were identified mainly in the peripheral areas (74.07\%) rather than in the central areas (25.93\%). Enlargement of mediastinal lymph nodes and pleural effusion may also occur, although this appears to be more likely in immunocompromised patients rather than in immunocompetent ones $(26,27)$.

Based on the clinical practice guidelines for the management of cryptococcal disease, initially published in 2000 (32) and updated in 2010 (33), it is essential to assess the hosts' immune status and the anatomical sites of involvement in patients with pulmonary cryptococcosis. For immunocompetent patients without symptoms, observation alone is required since pulmonary cryptococcosis may resolve spontaneously. When the lesions are enlarged and symptoms develop during the follow-up period, timely antifungal treatment is required. The preferred treatment regimens include oral azole therapy (fluconazole or itraconazole) for 6-12 months. In the present study, 3 asymptomatic cases did not receive any antifungal therapies but were observed. After 9 months, all patients had complete resolution of radiographic findings. Among the 23 patients who received antifungal therapy, significant improvement or resolution of radiographic findings was observed. In addition, 1 patient did not receive antifungal treatment due to rapidly progressing pancreatic cancer and succumbed to pancreatic cancer during the first 3-month follow-up.

In conclusion, pulmonary cryptococcosis in immunocompetent patients may be associated with environmental fungal exposure. The most common $\mathrm{CT}$ findings were multiple pulmonary nodules with poorly defined margins. Other findings included consolidation, GGO and mass. The majority of lesions involved the periphery of the lower lung lobes with a halo sign, air-bubble sign, air bronchogram and cavity. On contrast-enhanced chest CT scans, the nodules presented ring enhancement, and masses demonstrated inhomogeneous enhancement. Therefore, familiarity with the CT findings and occupational environment exposure history will assist in earlier and easier diagnosis of pulmonary cryptococcosis in immunocompetent patients.

\section{Acknowledgements}

This study was supported by grants from the National Natural Science Foundation of China (no. 81571707,81071182), the Program for Training Young Talents of Fujian Health (no. 2014-ZQN-ZD-35), the Natural Science Foundation of Fujian (no. 2015J01519), the Technology Foundation for Selected Overseas Chinese Scholar, the Ministry of Human Resources and Social Security of China (no. 2014-240) and the Scientific Research Foundation for the Returned Overseas Chinese Scholars, Ministry of Education of China (no. 2014-1685).

\section{References}

1. Smith IM, Stephan C, Hogardt M, Klawe C, Tintelnot K and Rickerts V: Cryptococcosis due to Cryptococcus gattii in Germany from 2004-2013. Int J Med Microbiol 305: 719-723, 2015.

2. Teodoro VL, Gullo FP, Sardi Jde C, Torres EM, Fusco-Almeida AM and Mendes-Giannini MJ: Environmental isolation, biochemical identification, and antifungal drug susceptibility of Cryptococcus species. Rev Soc Bras Med Trop 46: 759-764, 2013. 
3. Fang W, Fa Z and Liao W: Epidemiology of Cryptococcus and cryptococcosis in China. Fungal Genet Biol 78: 7-15, 2015.

4. Yamakawa H, Yoshida M, Yabe M, Baba E, Okuda K, Fujimoto S, Katagi H, Ishikawa T, Takagi M and Kuwano K: Correlation between clinical characteristics and chest computed tomography findings of pulmonary cryptococcosis. Pulm Med 2015: 703407, 2015.

5. Alanio A, Desnos-Ollivier M and Dromer F: Dynamics of Cryptococcus neoformans-macrophage interactions reveal that fungal background influences outcome during cryptococcal meningoencephalitis in humans. MBio 2: 4 e00158-11, 2011.

6. Schelenz S, Barnes RA, Barton RC, Cleverley JR, Lucas SB, Kibbler CC, Denning DW; British Society for Medical Mycology: British Society for Medical Mycology best practice recommendations for the diagnosis of serious fungal diseases. Lancet Infect Dis 15: 461-474, 2015

7. Perfect JR, Dismukes WE, Dromer F, Goldman DL, Graybill JR, Hamill RJ, Harrison TS, Larsen RA, Lortholary O, Nguyen $\mathrm{MH}$, et al: Clinical practice guidelines for the management of cryptococcal disease: 2010 update by the infectious diseases society of america. Clin Infect Dis 50: 291-322, 2010.

8. Sider L and Westcott MA: Pulmonary manifestations of cryptococcosis in patients with AIDS: CT features. J Thorac Imaging 9: 78-84, 1994.

9. Brizendine KD, Baddley JW and Pappas PG: Predictors of mortality and differences in clinical features among patients with cryptococcosis according to immune status. PLoS One 8: e60431, 2013.

10. Kishi K, Homma S, Kurosaki A, Kohno T, Motoi N and Yoshimura K: Clinical features and high-resolution CT findings of pulmonary cryptococcosis in non-AIDS patients. Respir Med 100: 807-812, 2006

11. Fox DL and Müller NL: Pulmonary cryptococcosis in immunocompetent patients: CT findings in 12 patients. AJR Am J Roentgenol 185: 622-626, 2005.

12. Espinel-Ingroff A and Kidd SE: Current trends in the prevalence of Cryptococcus gattii in the United States and Canada. Infect Drug Resist 8: 89-97, 2015.

13. Guarner $\mathrm{J}$ and Brandt ME: Histopathologic diagnosis of fungal infections in the 21st century. Clin Microbiol Rev 24: 247-280, 2011.

14. Barton RC: Laboratory diagnosis of invasive aspergillosis: From diagnosis to prediction of outcome. Scientifica (Cairo) 2013: 459405, 2013.

15. Ye XD, Ye JD, Yuan Z, Li WT and Xiao XS: Dynamic CT of solitary pulmonary nodules: Comparison of contrast medium distribution characteristic of malignant and benign lesions. Clin Transl Oncol 16: 49-56, 2014.

16. Feng X, Yao Z, Ling B, et al: Analysis of the varieties, genotypes and mating types of 110 clinical cryptococcal isolates from China. Chin J Microbiol Immunol 28: 193-197, 2008.

17. Wu SY, Lei Y, Kang M, Xiao YL and Chen ZX: Molecular characterisation of clinical Cryptococcus neoformans and Cryptococcus gattii isolates from Sichuan province, China. Mycoses 58: 280-287, 2015.

18. Galanis E, Macdougall L, Kidd S, Morshed M; British Columbia Cryptococcus gattii Working Group: Epidemiology of Cryptococcus gattii, British Columbia, Canada, 1999-2007. Emerg Infect Dis 16: 251-257, 2010.
19. Xie X, Xu B, Yu C, Chen M, Yao D, Xu X, Cai X, Ding C, Wang $L$ and Huang X: Clinical analysis of pulmonary cryptococcosis in non-HIV patients in south China. Int J Clin Exp Med 8: 3114-3119, 2015

20. Xie LX, Chen YS, Liu SY and Shi YX: Pulmonary cryptococcosis: Comparison of CT findings in immunocompetent and immunocompromised patients. Acta Radiol 56: 447-453, 2015.

21. Yuchong C, Fubin C, Jianghan C, Fenglian W, Nan X, Minghui Y, Yalin S and Zhizhong Z: Cryptococcosis in China (1985-2010): Review of cases from Chinese database. Mycopathologia 173: 329-335, 2012

22. Chen YY and Lai CH: Nationwide population-based epidemiologic study of cryptococcal meningitis in Taiwan. Neuroepidemiology 36: 79-84, 2011.

23. Zhang Y, Li N, Zhang Y, Li H, Chen X, Wang S, Zhang X, Zhang R, Xu J, Shi J and Yung RC: Clinical analysis of 76 patients pathologically diagnosed with pulmonary cryptococcosis. Eur Respir J 40: 1191-1200, 2012.

24. Charlier C, Nielsen K, Daou S, Brigitte M, Chretien F and Dromer F: Evidence of a role for monocytes in dissemination and brain invasion by Cryptococcus neoformans. Infect Immun 77: 120-127, 2009.

25. Sorrell TC, Juillard PG, Djordjevic JT, Kaufman-Francis K, Dietmann A, Milonig A, Combes V and Grau GE: Cryptococcal transmigration across a model brain blood-barrier: Evidence of the Trojan horse mechanism and differences between Cryptococcus neoformans var. Grubii strain H99 and Cryptococcus gattii strain R265. Microbes Infect 18: 57-67, 2016.

26. Lee WY, Wu JT, Jeng CM, HSU CY, Liu JS, Wang YC, Wu CY, Chang HY, Huang JC, Yang CM and Kung CH: CT Findings of pulmonary cryptococcosis: A series of 12 cases. Chin J Radiol 30: 319-325, 2005.

27. Ye F, Xie JX, Zeng QS, Chen GQ, Zhong SQ and Zhong NS: Retrospective analysis of 76 immunocompetent patients with primary pulmonary cryptococcosis. Lung 190: 339-346, 2012.

28. Lindell RM, Hartman TE, Nadrous HF and Ryu JH: Pulmonary cryptococcosis: CT findings in immunocompetent patients. Radiology 236: 326-331, 2005.

29. Zinck SE, Leung AN, Frost M, Berry GJ and Müller NL: Pulmonary Cryptococcosis: CT and Pathologic Findings. J Comput Assist Tomogr 26: 330-334, 2002.

30. Lee YR, Choi YW, Lee KJ, Jeon SC, Park CK and Heo JN: CT halo sign: The spectrum of pulmonary diseases. Br J Radiol 78: 862-865, 2005.

31. Chang WC, Tzao C, Hsu HH, Lee SC, Huang KL, Tung HJ and Chen CY: Pulmonary cryptococcosis: Comparison of clinical and radiographic characteristics in immunocompetent andimmunocompromised patients. Chest 129: 333-340, 2006.

32. Saag MS, Graybill RJ, Larsen RA, Pappas PG, Perfect JR, Powderly WG, Sobel JD and Dismukes WE: Practice guidelines for the management of cryptococcal disease. Infectious Diseases Society of America. Clin Infect Dis 30: 710-718, 2000.

33. Perfect JR, Dismukes WE, Dromer F, Goldman DL, Graybill JR, Hamill RJ, Harrison TS, Larsen RA, Lortholary O, Nguyen MH, et al: Clinical practice guidelines for the management of cryptococcal disease: 2010 update by the infectious diseases society of America. Clin Infect Dis 50: 291-322, 2010. 